\begin{tabular}{|c|l|}
\hline Title & Tomographic reconstruction of picosecond acoustic strain propagation \\
\hline Author(s) & Tomoda, Motonobu; Matsuda, Osamu; Wright, Oliver B.; Li V oti, Roberto \\
\hline Citation & $\begin{array}{l}\text { A pplied Physics Letters, 90(041114) } \\
\text { https://doi.org/L0.1063/2432238 }\end{array}$ \\
\hline Issue Date & 2007-01-22 \\
\hline Doc URL & http://hdl.handle.net/2115/17178 \\
\hline Rights & Copyright $\odot 2007$ American Institute of Physics \\
\hline Type & article \\
\hline File Information & APL_90_041114.pdf \\
\hline
\end{tabular}

Instructions for use 


\title{
Tomographic reconstruction of picosecond acoustic strain propagation
}

\author{
Motonobu Tomoda, ${ }^{\text {a) }}$ Osamu Matsuda, and Oliver B. Wright \\ Department of Applied Physics, Graduate School of Engineering, Hokkaido University, Sapporo \\ 060-8628, Japan \\ Roberto Li Voti \\ Dipartimento di Energetica, Università degli Studi di Roma "La Sapienza," Via A. Scarpa 14, \\ 00161 Roma, Italy
}

(Received 18 October 2006; accepted 13 December 2006; published online 26 January 2007)

\begin{abstract}
By means of an ultrafast optical technique, picosecond acoustic strain pulses in a transparent medium are tomographically visualized. The authors reconstruct strain pulses in Au-coated glass from time-domain reflectivity changes as a function of the optical angle of incidence, with $\sim 1 \mathrm{ps}$ temporal and $\sim 100 \mathrm{~nm}$ spatial resolutions. () 2007 American Institute of Physics.
\end{abstract}

[DOI: $10.1063 / 1.2432238$ ]

Laser picosecond acoustics is well adapted to the investigation of thin films and nanostructures. ${ }^{1-5}$ Subpicosecond light pulses are typically used to generate and detect $\sim 10-1000 \mathrm{GHz}$ longitudinal acoustic strain pulses. The acoustic strain pulse shape depends on the optoacoustic generation and the acoustic propagation, related, respectively, to the electron and phonon scattering mechanisms. ${ }^{1,4,6,7}$ Measuring the strain pulse shape is therefore useful for studying these mechanisms. In some materials it is possible to measure the strain pulse shape near a free surface by monitoring either surface displacements through beam deflection or changes in the phase of the optical reflectance. ${ }^{2,8}$ However, no existing method can continuously monitor picosecond strain pulse shapes during propagation. Stimulated by progress in lower frequency acoustic tomography, ${ }^{9}$ we present a method to achieve this.

Our sample consists of a thin metal film (with complex dielectric constant $\varepsilon_{2}$ ) deposited on a transparent substrate (with real $\varepsilon_{1}$ ), both materials being isotropic. For $s$-polarized probe light incidence at angle $\theta$ from the substrate side, the optical amplitude reflection coefficient $r_{0}$ (also termed the optical reflectance) can be expressed as ${ }^{10}$

$$
r_{0}=\frac{\cos \theta-\sqrt{\varepsilon_{2} / \varepsilon_{1}-\sin ^{2} \theta}}{\cos \theta+\sqrt{\varepsilon_{2} / \varepsilon_{1}-\sin ^{2} \theta}} .
$$

If $\varepsilon_{1}$ is slightly perturbed by a (anisotropic) longitudinal strain distribution $\eta_{33}(z, t)\left(\sim 10^{-5}\right.$ here $)$ in the depth $(z)$ direction, the reflectance is changed: ${ }^{11-13}$

$$
\begin{aligned}
\frac{\delta r_{0}}{r_{0}}= & i \frac{\pi P_{12}}{\sqrt{\varepsilon_{1}} \lambda \cos \theta} \int_{0}^{\infty} \eta_{33}(z, t)\left[\exp \left(-i \frac{2 \pi \sqrt{\varepsilon_{1}} z \cos \theta}{\lambda}\right)\right. \\
& \left.+r_{0} \exp \left(i \frac{2 \pi \sqrt{\varepsilon_{1}} z \cos \theta}{\lambda}\right)\right]^{2} d z
\end{aligned}
$$

where variations in $\varepsilon_{2}$ and the effect of the finite substrate thickness have been ignored. Here, $\lambda$ is the (central) wavelength of the probe light, $P_{12}\left(=\partial \varepsilon_{1} / \partial \eta_{33}\right)$ is a photoelastic constant, and $z=0$ at the 1-2 interface. Equation (2), which generalizes the formula derived in the past for reflectance changes at normal incidence, ${ }^{1,11}$ is an example of an inhomo-

${ }^{a)}$ Electronic mail: mtomoda@eng.hokudai.ac.jp geneous Fredholm integral. ${ }^{14}$ We calculate $\eta_{33}(z, t)$ in the transparent substrate at a given time $t$ from the observed reflectivity variation $\delta R_{0} / R_{0}=2 \operatorname{Re}\left(\delta r_{0} / r_{0}\right)$ as a function of $\theta$ by solving the inverse problem (where $R_{0}=\left|r_{0}\right|^{2}$ ). ${ }^{14,15}$ (A similar approach involving optical phase variations should be feasible.)

A polished $10 \mathrm{~mm}$ radius hemisphere of BK7 glass, whose flat surface is coated with a thin polycrystalline $\mathrm{Au}$ film of thickness of $600 \mathrm{~nm}$, is mounted on a rotation stage to set $\theta$ (see Fig. 1). A photodetector is mounted on a separate coaxial rotation stage set to $2 \theta$. Visible $s$-polarized pump light pulses of duration of $\sim 100 \mathrm{fs}$, repetition rate of $82 \mathrm{MHz}$, wavelength of $415 \mathrm{~nm}$, and pulse energy of $1.1 \mathrm{~nJ}$ from the second harmonic of a Ti:sapphire mode-locked laser are used to illuminate an $\sim 20 \mu \mathrm{m}$ diameter [full width at half maximum (FWHM) intensity at $\theta=0^{\circ}$ ] spot on the center of the flat Au-coated face of the hemisphere from the air side. This produces a maximum temperature rise of $\sim 20^{\circ} \mathrm{C}$ (in $\mathrm{Au}$ ) after pump pulse arrival and a maximum steady state rise of $\sim 110^{\circ} \mathrm{C}$ at the $\mathrm{BK} 7-\mathrm{Au}$ interface in the pump beam spot. (After optical absorption to a depth $\zeta=18 \mathrm{~nm}$, electron diffusion distributes the initial temperature rise to depths of $\sim 10 \zeta^{4,16}$ ) The thermoelastically generated acoustic strain

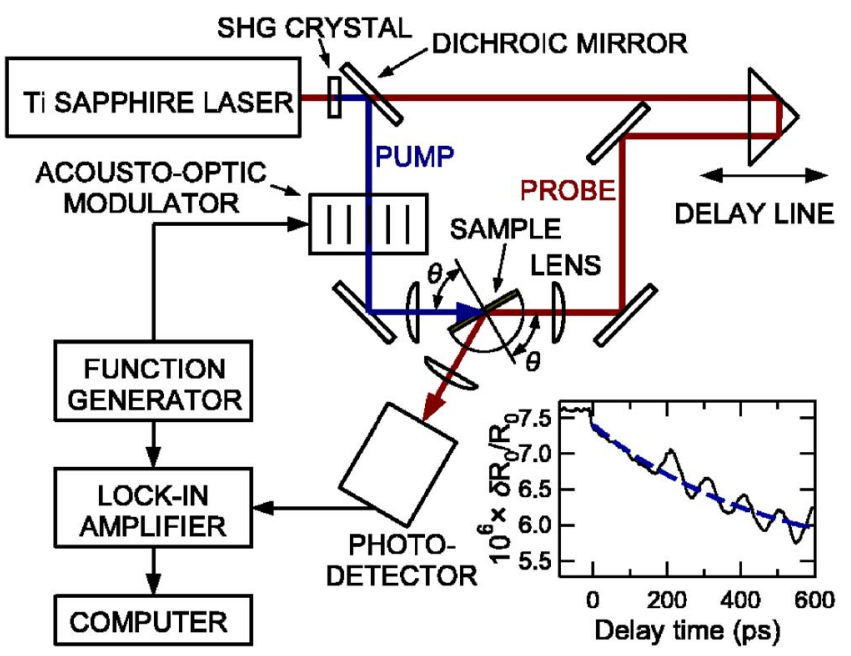

FIG. 1. (Color online) Experimental setup. Inset: relative reflectivity change for $\theta=60^{\circ}$ (solid line) and exponential fit to thermoreflectance (dashed line). SHG: second-harmonic generation crystal. 

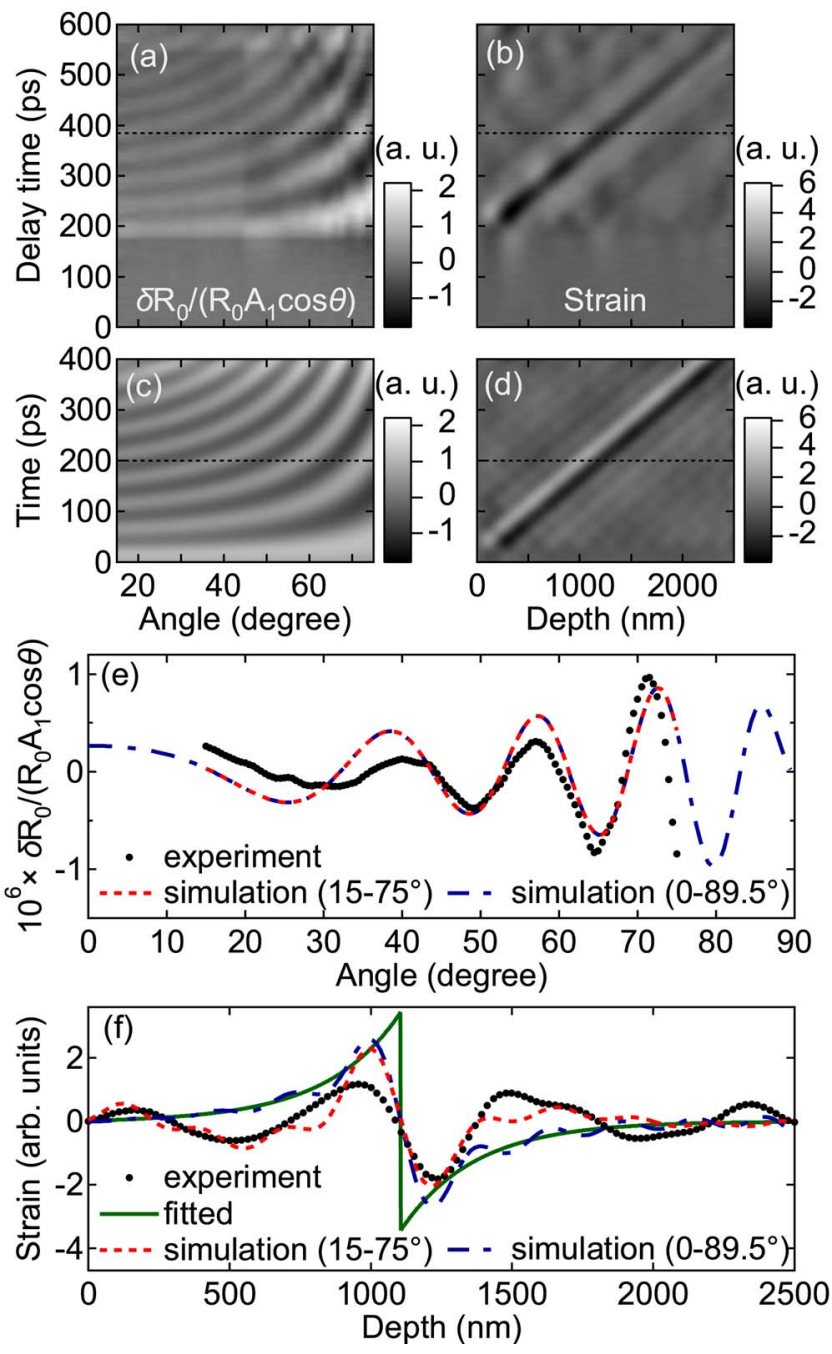

FIG. 2. (Color online) (a) Density plot of the measured relative reflectivity variation $\delta R_{0} / R_{0}$, normalized by $A_{1} \cos \theta$. (b) Experimentally reconstructed strain $\eta_{33}(z, t)$. (c) Simulated $\delta R_{0}(t) / R_{0}$ (for constant absorbed fluence). (d) Theoretically reconstructed $\eta_{33}(z, t)$. (e) $\delta R_{0} /\left(R_{0} A_{1} \cos \theta\right)$ at $385 \mathrm{ps}$ vs angle $\theta$. (f) Reconstructed strain distributions at $t=385 \mathrm{ps}$. In (e) and (f) the dots are experimental data, the dashed lines are fits using the same range of $\theta$ as in experiment, and the dotted-dashed lines are fits using angles $0^{\circ}-89.5^{\circ}$.

pulses are bipolar in shape, with an amplitude of $\sim 10^{-5}$, duration of $\sim 100 \mathrm{ps}$, spatial extent of $\sim 300 \mathrm{~nm}$ (in $\mathrm{Au}$ ), and with frequency components of up to $\sim 10 \mathrm{GHz} .{ }^{4}$ Infrared $(\lambda=830 \mathrm{~nm}) s$-polarized probe pulses pass through an optical delay line and are focused to an $\sim 10 \mu \mathrm{m}$ diameter (FWHM intensity at $\theta=0^{\circ}$ ) spot on the Au film from the substrate side. $\delta R_{0} / R_{0}$ is monitored at the photodetector as a function of $\theta$ (in the range of $15-75^{\circ}$ ) and the pump-probe delay time $t$. By chopping the pump beam at $2 \mathrm{MHz}$ and using lock-in detection, we track $\delta R_{0}(t) / R_{0}\left(\sim 10^{-5}\right)$. Both optical beams are incident at angle $\theta$; the optical spots are thus ellipses with the same aspect ratio, whose horizontal sizes depend on $\theta$.

The inset in Fig. 1 shows data for $\delta R_{0}(t) / R_{0}$ at $\theta=60^{\circ}$. At $t=185 \mathrm{ps}$ the center of the strain pulse generated in the $\mathrm{Au}$ arrives at the $\mathrm{Au}-\mathrm{BK} 7$ interface (i.e., when $t=d / v_{2}$, where $d=600 \mathrm{~nm}$ and $v_{2}=3360 \mathrm{~m} \mathrm{~s}^{-1}$ are the Au film thickness and longitudinal sound velocity $\left.{ }^{17}\right) . \delta R_{0}(t) / R_{0}$ then oscillates owing to the photoelastic effect: the strain pulse acts as a moving mirror, producing oscillations by interference between the probe light reflected from the $\mathrm{Au}$ and from the

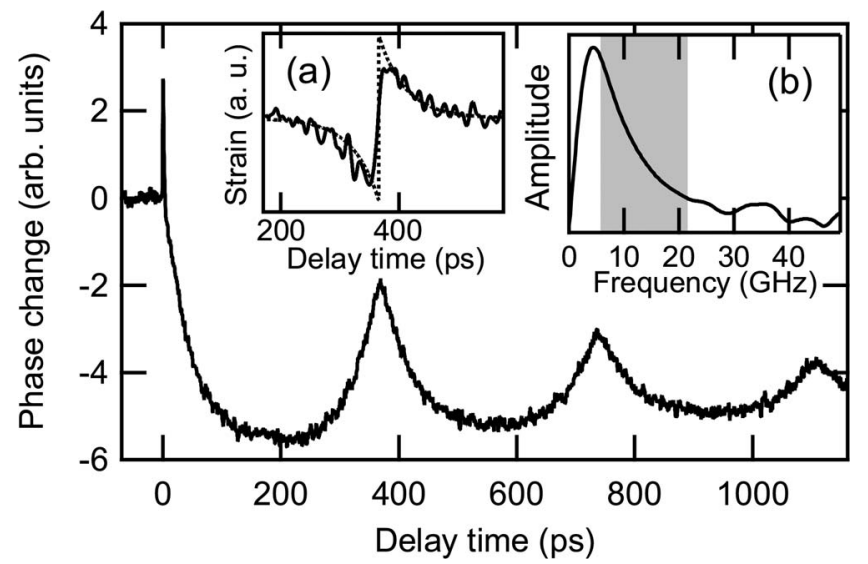

FIG. 3. Optical phase change measured interferometrically, showing multiple acoustic echoes. Inset (a): time derivative of the phase change, proportional to the acoustic strain pulse shape for the first echo. Dotted line: fit. Inset (b): Fourier spectrum of the strain in (a). The shaded range corresponds to the angle-scanned experiment.

strain pulse. ${ }^{3}$ The period is $\tau=\lambda /\left(2 n_{1} v_{1} \cos \theta\right) \approx 94$ ps for $\theta=60^{\circ}$ and $n_{1}=\sqrt{\varepsilon_{1}}=1.51$ (the refractive index of BK7), ${ }^{18}$ where $v_{1}=5860 \pm 30 \mathrm{~m} \mathrm{~s}^{-1}$ is the derived longitudinal sound velocity of BK7 (from a range of $\theta$ values), close to the literature value. ${ }^{18}$ [In fact if $\eta_{33}(z, t)=\eta_{33}\left(z-v_{1} t\right)$, one can show that Eq. (2) contains a term proportional to $(C / \cos \theta) \cos \left(2 \pi t / \tau+\phi_{0}\right)$, where $C$ and $\phi_{0}$ are constants for given materials and $\lambda$.] On transmission to the BK7 the acoustic strain pulse length increases by a factor of $v_{1} / v_{2}$ $=1.75$.

The background thermoreflectance variation is subtracted by fitting to an exponential function (the dashed line in Fig. 1 inset). The data are then divided by $A_{1} \cos \theta$ to account for the variation with $\theta$ of the absorbed pump fluence (arising in part from the elongation of the elliptical pump spot as $\theta$ increases), where $A_{1}(\theta)=1-R_{1}(\theta)$ is calculated from the pump beam reflectivity $R_{1}$ using known optical constants. ${ }^{16}$ The increase in the (elliptical) probe spot area with $\theta$ ensures that the relative sizes of the pump and probe spots remain constant. Figure 2(a) shows a resulting density plot, corresponding to 116 different angles: $15^{\circ}-20^{\circ}$ in steps of $1^{\circ}$, and $20.5^{\circ}-75^{\circ}$ in steps of $0.5^{\circ}$. Angles $<15^{\circ}$ or $>75^{\circ}$ are precluded by lens overlap. The expected increase in $\tau$ with $\theta$ is clearly seen. Some random and systematic noise owing to slight optical alignment errors are evident. ${ }^{19}$ A cross section of these data at delay time $t$ $=385 \mathrm{ps}$ is shown by the dots in Fig. 2(e).

In order to find $\eta_{33}(z, t)$, the whole $\theta-t$ data set was processed by the singular value decomposition method, ${ }^{14,15,20}$ yielding a set of singular functions with an optimized eigenvalue number $N=12$. The diagonal line in Fig. 2(b) shows the resulting reconstructed strain pulse $\eta_{33}(z, t)$, taking $\varepsilon_{1}$ from the literature. ${ }^{16}$ We derive $v_{1}=5870 \pm 10 \mathrm{~m} \mathrm{~s}^{-1}$ from these data (consistent with $v_{1}$ above). A cross section of these data at time $t=385$ ps is shown by the dots in Fig. 2(f).

We have used a separate technique to determine $\eta_{33}(z, t)$ in the Au: a Sagnac interferometer measures the outward displacement $u(t)$ of the free surface of the Au film through optical phase changes $\delta \phi(t)$ under identical optical excitation conditions. ${ }^{8,21}$ The acoustic strain pulse in the Au varies $\propto$ $-d u(t) / d t^{2,8} \delta \phi(t)$ is shown in Fig. 3, characterized by several echoes decaying in height according to the acoustic 
strain reflection coefficient $(\sim 0.6)$ at the Au/BK7 interface. The temporal derivative of the first echo is shown in the inset (a). Because of the relatively small ultrasonic attenuation in polycrystalline $\mathrm{Au}$ and in silica at the acoustic frequencies $(\sim 10 \mathrm{GHz})$ and propagation distances $(<3 \mu \mathrm{m})$ in question, ${ }^{4,22}$ we directly compare this bipolar pulse shape with the acoustic strain pulse shape measured tomographically, accounting for the above-mentioned factor of $v_{1} / v_{2}$ $=1.75 .^{23}$ The dotted line in the inset (a) of Fig. 3 is a fit to the measured strain pulse in the Au based on exponential tails [in the form $\operatorname{sgn}\left(t^{\prime}\right) \exp \left(-v_{2}\left|t^{\prime}\right| / L\right)$, where $L=150 \mathrm{~nm}$ and $t^{\prime}=t$ $-t_{0}$, where $t_{0}$ corresponds to the center of the strain pulse]. The equivalent strain pulse in the BK7 in the spatial domain [in the form $-\operatorname{sgn}\left(z^{\prime}\right) \exp \left(-\left|z^{\prime}\right| / l\right)$, where $l=\left(v_{1} / v_{2}\right) L$ $=260 \mathrm{~nm}$ and $\left.z^{\prime}=v_{1} t^{\prime}\right]$ is shown by the solid line in Fig. 2(f). Compared with the tomographic reconstruction, this strain pulse is significantly sharper.

To pin down the reasons for this we first plot in Fig. 2(c) the prediction for $\delta R_{0}(\theta, t) / R_{0}$ (at constant absorbed fluence), calculated from Eq. (2) using a single fitted strain pulse (appropriate for our temporal range $t<600 \mathrm{ps}$ ). The time origin corresponds to the strain pulse arrival at the $\mathrm{Au}-$ BK7 interface $(t=185 \mathrm{ps})$. A cross section corresponding to $t=385 \mathrm{ps}$ in experiment is shown by the dashed line in Fig. 2(e), giving reasonable agreement with experiment. The corresponding reconstructed $\eta_{33}(z, t)$ for $\theta=15^{\circ}-75^{\circ}$ is shown in Fig. 2(d), and a cross section at $t=385$ ps is shown by the dashed line in Fig. 2(f) (for $N=14$ ). The resulting strain pulse shape is similar to the experimental one. We have also plotted by the dotted-dashed lines in Figs. 2(e) and 2(f) the predictions corresponding to the angle range of $0^{\circ}-89.5^{\circ}$ (for $N=18$ ). The reconstruction is only slightly improved by this.

These findings can be understood by considering the constraints on the reconstructed acoustic frequencies. A measurement at angle $\theta$ is sensitive to an acoustic strain Fourier component at a frequency $f=1 / \tau=\left(2 n_{1} v_{1} \cos \theta\right) / \lambda$; our measurements for angles $15^{\circ}-75^{\circ}$ correspond to the limited range of $5.5-20.7 \mathrm{GHz}$, as shown by the shaded region in the inset (b) of Fig. 3 together with the temporal Fourier transform of the interferometrically measured strain pulse. For angles $0^{\circ}-$ $89.5^{\circ}$, this frequency range broadens to $0.2-22 \mathrm{GHz}$. It is primarily the frequency range that limits the reconstruction in our case. Apart from noise reduction, the best way to improve our reconstruction fidelity would therefore be to increase the maximum frequency $f$ by reducing the optical wavelength $\lambda$ (or by using a $\lambda$ scan involving smaller $\lambda$ ).

Our reconstruction method also relies on the use of literature values of optical constants to correct for the $A_{1}(\theta)$ variation. Ideally this measurement should be done experimentally. Another experimental limitation is the possible walk-off between the strain distribution and the obliquely incident optical probe beam in the substrate as the strain penetrates deeper. We have made quantitative estimates of this effect by taking the cross correlation of the probe beam and strain pulse lateral distributions. In the present experiment this effect should only decrease the signal by a maximum of $8 \%$ at $t=600$ ps and $\theta=75^{\circ}$.

The spatial resolution in the present study is limited to $\sim 100 \mathrm{~nm}$, but it should be possible to improve on this using a shorter optical probe wavelength. The method could be extended to reconstruct other fields that couple to the dielectric constant, such as carrier or temperature distributions or nonlinear optical pulse propagation, in transparent or semitransparent materials.

${ }^{1}$ C. Thomsen, H. T. Grahn, H. J. Maris, and J. Tauc, Phys. Rev. B 34, 4129 (1986).

${ }^{2}$ O. B. Wright and K. Kawashima, Phys. Rev. Lett. 69, 1668 (1992).

${ }^{3}$ H.-N. Lin, R. J. Stoner, H. J. Maris, and J. Tauc, J. Appl. Phys. 69, 3816 (1991).

${ }^{4}$ O. B. Wright, Phys. Rev. B 49, 9985 (1994).

${ }^{5}$ C. Rossignol and B. Perrin, IEEE Trans. Ultrason. Ferroelectr. Freq. Control 52, 1354 (2005).

${ }^{6}$ G. Tas and H. J. Maris, Phys. Rev. B 49, 15046 (1994).

${ }^{7}$ O. B. Wright, B. Perrin, O. Matsuda, and V. E. Gusev, Phys. Rev. B 64, 081202 (2001).

${ }^{8}$ D. H. Hurley and O. B. Wright, Opt. Lett. 24, 1305 (1999).

${ }^{9}$ P. Gren, S. Schedin, and X. Li, Appl. Opt. 37, 834 (1998).

${ }^{10}$ B. E. A. Saleh and M. C. Teich, Fundamentals of Photonics (Wiley, New York, 1991), p. 205.

${ }^{11}$ O. Matsuda and O. B. Wright, J. Opt. Soc. Am. B 19, 3028 (2002).

${ }^{12}$ O. Matsuda and O. B. Wright, Rev. Sci. Instrum. 74, 895 (2003).

${ }^{13} \mathrm{O}$. Matsuda and O. B. Wright (unpublished).

${ }^{14}$ J. F. Power, Rev. Sci. Instrum. 73, 4057 (2002).

${ }^{15}$ C. Glorieux, R. Li Voti, J. Thoen, M. Bertolotti, and C. Sibilia, Inverse Probl. 15, 1149 (1999).

${ }^{16}$ E. D. Palik, Handbook of Optical Constants (Academic, New York, 1985), Vol. I, p. 294.

${ }^{17}$ O. L. Anderson, in Physical Acoustics, edited by W. P. Mason (Academic, New York, 1965), Vol. 3B, p. 43.

${ }^{18}$ See http://www.ispoptics.com/new_webpage/opt_mat_specs.htm

${ }^{19}$ The pump and probe spot overlap was optimized for each angle, and the photodetector position was readjusted for $\theta>45^{\circ}$.

${ }^{20}$ R. Li Voti, C. Sibilia, and M. Bertolotti, Int. J. Thermophys. 26, 1833 (2005).

${ }^{21}$ T. Tachizaki, T. Muroya, O. Matsuda, Y. Sugawara, D. H. Hurley, and O. B. Wright, Rev. Sci. Instrum. 77, 043713 (2006).

${ }^{22}$ T. C. Zhu, H. J. Maris, and J. Tauc, Phys. Rev. B 44, 4281 (1991).

${ }^{23}$ Because of the limitation on spatial resolution in the reconstructed strain pulses, the effects of ultrasonic attenuation were not included in the numerical modeling. 